\title{
Nanotheranostics
}

2018; 2(2): 106-116. doi: 10.7150/ntno.19379

Research Paper

\section{Multifunctional PEGylated Albumin//R780/Iron Oxide Nanocomplexes for Cancer Photothermal Therapy and MR Imaging}

\author{
Ssu-Yu Lin, Rih-Yang Huang, Wei-Chen Liao, Chun-Chiao Chuang and Chien-Wen Chang ${ }^{\bowtie}$ \\ Department of Biomedical Engineering and Environmental Sciences, National Tsing Hua University, Hsinchu 30013, Taiwan, R.O.C. \\ $\triangle$ Corresponding author: Tel.: 886-3-5715131 ext. 35531. Fax: 886-3-5718649. Email: chienwen@mx.nthu.edu.tw \\ (C) Ivyspring International Publisher. This is an open access article distributed under the terms of the Creative Commons Attribution (CC BY-NC) license \\ (https://creativecommons.org/licenses/by-nc/4.0/). See http://ivyspring.com/terms for full terms and conditions.
}

Received: 2017.01.28; Accepted: 2017.08.25; Published: 2018.01.01

\begin{abstract}
A multifunctional albumin/superparamagnetic iron oxide nanoparticle (SPIO) nanocomplex system to deliver IR780, a photothermal agent, for cancer theranostic applications was proposed in this study. Single emulsion method was utilized to fabricate the human albumin/IR780/SPIO (HISP) nanocomplexes with a hydrophobic core (SPIO and IR780) and a hydrophilic shell (human serum albumin (HSA) and poly (ethylene glycol) (PEG)). Effects of PEGylation on the size and surface potential of nanocomplexes were analyzed. Nanospheres containing uniformly dispersed SPIO was observed using Transmission Electron Microscopy (TEM) imaging. As a potential magnetic resonance (MR) imaging agent, the HISP displayed dose-dependent T2-weighted imaging contrast $\left(R 2=81.6 \mathrm{mM}^{-1} \mathrm{~s}^{-1}\right)$. Good colloidal stability was verified from the nanocomplexes under difference circumstances. The nanocomplexes were taken up by cancer cells efficiently and led to significant photothermal-mediated cancer cell death upon short-term near infrared (NIR) irradiation in vitro. Via intravenous injection, PEG-HISP can efficiently deliver IR780 to tumor sites and showed strong photothermal effect compared to free drug on the mice model. Significant tumor suppression by the photothermal treatments using PEG-HISP was demonstrated from the mice CT26 xenograft model. Good safety profile of the PEG-HISP was confirmed from histological examination and liver functional analysis. Taken together, the results suggest that PEG-HISP is a safe and robust nano-theranostic platform for advanced anti-cancer treatment.
\end{abstract}

Key words: photothermal therapy, IR780, superparamagnetic iron oxide nanoparticles, magnetic resonance imaging, colon cancers

\section{Introduction}

Hyperthermia treatment has been utilized as an effective anti-cancer approach with a long history[1]. Compared to normal tissues, cancer cells are highly heat-sensitive due to the impaired vasculature and hypoxic microenvironment in tumor region[2, 3]. Heat could effectively induce cancer cell apoptosis via activating cellular caspase signaling pathway[4-6]. Meanwhile, the therapeutic efficacy of conventional hyperthermic methods for cancer ablation is quite limited because of poor and inhomogenous heat delivery to tumor regions. To tackle the above challenges, nanomaterial-based photothermal therapy integrating with drug delivery features have emerged as a more controllable and effective solution[7-9]. It has been previously shown that light within the near infrared (NIR) window (650 - $900 \mathrm{~nm})$ exhibited greatest tissue penetration capability[10]. Consequently, tissue-penetrable NIR-sensitive dyes are often utilized as the photo-responsive agents to generate effects of hyperthermia [11-14], photoacoustics $[15,16]$ or photoluminescence[17] within the whole tumor region.

IR780 is a hydrophobic dye with maximum absorption at $780 \mathrm{~nm}$, which could convert the NIR photons into heat and reactive oxygen species (ROS) and has been attempted for both photothermal and 
photodynamic therapy[12]. Compared to indocyanine green (ICG), IR780 exhibits better stability and higher fluorescent intensity due to its rigid chemical structure[18, 19]. Due to its hydrophobicity and short circulation time, IR780 requires suitable delivery formulation for its further in vivo applications[20-22]. Albumin, one of the most abundant serum proteins, has received great attention on hydrophobic drug delivery. The FDA-approved albumin-paclitaxel formulation, Abraxane ${ }^{\circledR}$, is perhaps the most well-known example of albumin-based delivery technology[23, 24]. Albumin is capable of penetrating vascular walls via gp60 receptor-mediated transcytosis in the endothelial cells[24]. Besides, nano-sized albumin particles could accumulate in tumors via Enhanced Permeation and Retention (EPR) effect[25].

Combination of biopolymer and inorganic nanoparticles is an attractive strategy to prepare nanocomplexes with multiple theranostic functionalities. For example, further incorporation of superparamagnetic iron oxide nanoparticles (SPION) affords drug delivery carriers with magnetic resonance (MR) imaging capability[26, 27] or magnetic-guided drug delivery[28]. It has been shown that surface decoration with albumin could improve the in vivo stability of SPIO for better magnetic resonance imaging (MRI)[29]. In this study, we proposed a human albumin/IR780/SPIO nanocomplexes (HISP) system capable of photothermal therapy and molecular imaging. This nano-delivery platform mainly utilizes FDA-approved biomaterials, including: human serum albumin (HSA), superparamagnetic iron oxide nanoparticles (SPION) and polyethylene glycol (PEG).

The HISP was prepared by emulsifying hydrophobic SPIO and IR780 in HSA aqueous solution in single step. Size, morphology and surface potential of the HISP were characterized using ZetaSizer and Transmission Electron Microscope (TEM). Colloidal stability of the PEG-HISP at serum free or serum-containing media was examined. In vitro photothermal effects of the PEG-HISP were tested on mouse colon cancer cells (CT26). T2 relaxivity and in vivo MR imaging of the PEG-HISP were determined using a 7T MRI. As a proof of concept, in vivo toxicity and therapeutic efficacy of the HISP were studied on a mouse colon cancer (CT26) model.

\section{Experimental Section}

\section{Materials}

Iron (III) chloride, Trioctylphosphine oxide (TOPO) and paraformaldehyde were purchased from
Alfa Aesar (Ward Hill, MA, USA). Sodium oleate was purchased from TCI (Tokyo Kasei Kogyo, Inc). MTT was purchased from MDBio, Inc. (Taipei, Taiwan). Dulbecco's Modified Eagles' Medium (DMEM) and Dulbecco's phosphate buffered saline (DPBS) were purchased from Gibco (Carlsbad, CA, USA). Penicillin-streptomycin (P/S) and cosmic calf serum (CCS) were purchased from Hyclone (Logan, UT, USA). Human serum albumin (HSA), oleic acid and IR-780 iodide were purchased from Sigma-Aldrich. mPEG5k-MAL was obtained from Jenkem. 2-iminothiolane (Traut's Reagent) was bought from ThermoScientific. SPION was synthesized as previously described[30].

\section{Synthesis of HISP}

$150 \mathrm{mg}$ of HSA were dissolved in $5 \mathrm{ml}$ deionized water with constant stirring. IR-780 $(5 \mathrm{mg} / \mathrm{ml})$ and SPION $(20 \mathrm{mg} / \mathrm{ml})$ were dissolved in chloroform before adding drop by drop into the HSA solution with stirring all the time. The obtained green dispersions were ultrasonicated at $20 \%$ amplitude of the maximum power for 150 seconds by Sonics VCX $750 \mathrm{~W}$ probe sonication (Sonics, Newtown, CT, USA; maximum power: $750 \mathrm{~W}$; frequency: $20 \mathrm{kHz}$; probe diameter: $13 \mathrm{~mm}$ ). Following processed on a rotary evaporator under the reduced pressure to remove the remaining chloroform, the samples were purified to remove free HSA using an ultrafiltration membrane (molecular weight cut-off (MWCO) $100 \mathrm{kDa}$ ). Finally, the samples were filtered through $0.22 \mu \mathrm{m}$ filter membrane, and magnetic purified overnight at $4{ }^{\circ} \mathrm{C}$. Magnetic purification was performed by placing a permanent magnet besides the wall of bottle containing the synthesized nanoparticle suspension (as shown in Figure 5A). To maximize the recovery of magnetic HISP, the magnetic attraction process usually performed overnight. The attracted HISP was washed with PBS then resuspended with DI $\mathrm{H}_{2} \mathrm{O}$. Albumin/SPIO (HSP) and albumin/IR780 (HIP) nanocomplexes was prepared using same procedure as described above except without adding IR780 or SPIO respectively.

\section{Encapsulation Efficiency (E.E.) and Loading efficiency (L.E.) of IR780 in HISP}

Lyophilized HISP was reconstituted in DMSO. Amount of IR780 was calculated by a IR780 standard curve using a UV-Vis spectrophotometer. E.E. and L.E. of IR780 in the HISP were calculated using the following equations:

E.E. of IR780 $(\%)=($ Mass of total encapsulated IR780/(Initial amount of feeding IR780)* $100 \%$

L.E. of IR780 $(\%)=($ Mass of IR780 in HISP) $/$ (Mass of HISP) ${ }^{\star} 100 \%$ 


\section{Encapsulation Efficiency (E.E.) and Loading efficiency (L.E.) of SPIO in HISP}

Amount of SPIO in HISP was quantitative using KSCN method. In brief, the nanoparticles were dissolved in a $12 \mathrm{~N} \mathrm{HCl}$ solution, afterward, the ferrous ions were oxidized by $30 \%$ ammonia persulfate in $65^{\circ} \mathrm{C}$ oven for 30 minutes. The ferric ions were reacted with $5 \% \mathrm{KSCN}$ to produce red $\mathrm{Fe}[\mathrm{SCN}]^{2+}$ solution. Amount of SPIO was determined by measuring the absorbance at $480 \mathrm{~nm}$ and calculated using a standard curve. E.E. and L.E. of SPIO in the HISP were calculated using the following equations:

E.E. of SPIO $(\%)=($ Mass of total encapsulated

SPIO/(Initial amount of feeding SPIO) ${ }^{*} 100 \%$

L.E. of SPIO $(\%)=($ Mass of SPIO in HISP $) /($ Mass of HISP) ${ }^{*} 100 \%$

\section{Synthesis of PEG-HISP}

The primary amines on the surface of HISP were thiolated by reacting with 2-iminothiolane (Traut's Reagent; $10 \mathrm{mg} / \mathrm{mL}$, approximately 10 equiv to an HSA molecule) in PBS ( $\mathrm{pH} 7.4$ ) at room temperature for 30 minutes. The thiolated HISP was concentrated using a Centricon-100 concentrator (Amicon Ultra-4 and -15 Centrifugal Filter Units-100000 MWCO, Millipore) to remove free 2-iminothiolane. The thiolated HSA was quickly transferred to react with mPEG-MAL at various HSA:PEG ratios (1:0; 1:10; 1:50; 1:200) in PBS (pH 7.4), and the reaction was allowed to proceed at room temperature for 6 hours. The resultant mixture was concentrated using a Centricon30 concentrator and stored at $4{ }^{\circ} \mathrm{C}$ before further usage.

\section{Physicochemical characterizations of PEG-HISP}

The PEG-HISP was dissolved in distilled water. Particle size and zeta potential of the nanocomplexes were measured using a Zetasizer (Nano Series, Malvern, UK). Transmission Electron Microscopy (TEM, JEOL JEM-1200EX II, Japan) was used to characterize the morphology of PEG-HISP. Ultraviolet-visible (UV-vis) spectra of the PEG-HISP were recorded using a spectrophotometer (Thermo scientific, NANO DROP 2000c spectrophotometer). T2-weighted images were studied by 7T MRI system (Bruker biospec 70/30 MRI, USA).

\section{IR780 release from PEG-HISP}

Cumulative release of IR780 from the PEG-HISP was performed. Predetermined amount of PEG-HISP were dissolved in $1 \mathrm{~mL}$ of $\mathrm{DI} \mathrm{H}_{2} \mathrm{O}$ or PBS (pH 7.4) and transferred to a dialysis bag (MWCO 12kDa to
$14 \mathrm{kDa}$ ). The dialysis bag was immerged in $13 \mathrm{~mL}$ release medium containing $0.1 \%$ tween 80 and kept in $37^{\circ} \mathrm{C}$ with stirring. After the predesignated time points (1h, $4 \mathrm{~h}, 24 \mathrm{~h} \mathrm{...} \mathrm{168h),} \mathrm{the} \mathrm{release} \mathrm{medium} \mathrm{was}$ withdrawn, lyophilized, and reconstituted in DMSO. Amount of the released IR780 was determined using a fluorescence spectroscopy (emission: $820 \mathrm{~nm} \mathrm{/}$ excitation:780 $\mathrm{nm}$ ).

\section{Photothermal properties of PEG-HISP}

PEG-HISP was dissolved in deionized water and subjected to laser irradiation $\left(1 \mathrm{~W} / \mathrm{cm}^{2}\right)$ at $808 \mathrm{~nm}$. Temperature of the sample solutions was recorded using a Mini thermal imager (Avio NEC Thermo Shot F30W).

\section{Cell culture}

The CT26 (murine colon adenocarcinoma) cells were cultured in DMEM supplemented with $10 \%$ CCS and $1 \mathrm{x} \mathrm{P} / \mathrm{S}$ maintained at $37{ }^{\circ} \mathrm{C}$ and $5 \% \quad \mathrm{CO}_{2}$ atmosphere. The cells were subcultured at a split ratio of 1:4 while cell confluency reached $80 \%$.

\section{Cellular uptake}

The CT26 cells were seeded in the 24 -well plates at density of $5 \times 10^{4}$ cells per well overnight. The cells were incubated with PEG-HISP $(1 \mu \mathrm{g} / \mathrm{mL})$ for various time lengths. Following by the incubation, the cells were washed twice with PBS then collected by trypsinization. Cellular fluorescence was measured on a flow cytometer (BD FACSVerse ${ }^{\mathrm{TM}}$, BD Biosciences, San Jose, CA) with $633 \mathrm{~nm}$ excitation and $780 \mathrm{~nm}$ emission for IR780. The cells without treatment were used a control group in the cellular uptake experiment.

\section{Cell viability assay}

CT26 cells were seeded in the 96-well plates at density of $5 \times 10^{3}$ cells per well overnight then received various concentrations of IR780 (dissolved in DMSO), HISP and PEG-HISP. After incubation for 24 hours, the culture medium was removed and the cells were washed with PBS. MTT $(0.5 \mathrm{mg} / \mathrm{ml})$ in fresh medium was added to each well following by incubation at $37^{\circ} \mathrm{C}$ and $5 \% \mathrm{CO}_{2}$ atmosphere for 4 hours. Finally, following remove the medium, each well was added with DMSO and measured at $580 \mathrm{~nm}$ using a microplate reader.

\section{In vitro and in vivo $M R$ imaging}

T2-weighted images of HISP or tumor sections were performed by 7T MR imaging system (Bruker biospec 70/30 MRI, USA). To measure the spin-spin relaxation times (T2), ferric iron concentrations of HISP at $1,0.5,0.25,0.125$, and 0.0625 (mM Fe) were prepared separately in deionized water and deposited 
into $250 \mu \mathrm{L}$ PCR tubes. A multislice multiecho (MSME)-T2 map pulse sequence was performed using the following parameters: TR/TE $=3000 \mathrm{~ms} / 11 \mathrm{~ms}$ (32 fitted echoes in $11 \mathrm{~ms}$ intervals), matrix size $=256$ $\times 256, \mathrm{FOV}=6 \times 6 \mathrm{~mm}$, and NEX $=3$. The T2 weighted images of HISP were shown at the first echo times $(\mathrm{TR} / \mathrm{TE}=3000 \mathrm{~ms} / 11 \mathrm{~ms})$. For in vivo T2 weighted imaging, at 0 hour or 24 hours after intravenous injection of PBS or PEG-HISP, the mice were anesthetized by isoflurane, then both axial and coronal images of tumor sections were acquired by a rapid acquisition relaxation enhancement sequence (RARE) with the parameters as follows: TR/TE $=2500$ $\mathrm{ms} / 30 \mathrm{~ms}$, rare factor $=6$, matrix size $=256 \times 256$, slice thickness $=1 \mathrm{~mm}, \mathrm{FOV}=3.5 \mathrm{~mm} \times 3.5 \mathrm{~mm}$, interstice distance $=1 \mathrm{~mm}$ (10 slices) and NEX $=3$.

\section{In vitro photothermal effect}

CT26 colon adenocarcinoma cells were seeded in the 48 -well plates at density $2.5 \times 10^{4}$ cells per well overnight then treated with PEG-HISP $(0.01 \mathrm{mg} / \mathrm{ml})$. Subsequently, culture medium containing HISP was changed with fresh serum-free DMEM. $808 \mathrm{~nm}$ laser was used to irradiate $\left(1.5 \mathrm{~W} / \mathrm{cm}^{2}\right)$ on the cells for 1,3 or 5 minutes. After laser irradiation, the cells were continued to incubate for 2 hours. Next, the culture medium was replaced with fresh 10\% CCS/DMEM and incubated for 24 hours. Finally, the mixed solution consisting of MTT $(0.5 \mathrm{mg} / \mathrm{ml})$ in fresh medium was added in each well, and the cells were incubated for 4 hours at $37^{\circ} \mathrm{C}$ and $5 \% \quad \mathrm{CO}_{2}$ atmosphere. Finally, following remove the medium, each well was added DMSO and measured at $580 \mathrm{~nm}$ using a microplate reader.

\section{Animal model}

The animals were handled in accordance to the animal use protocol of the National Tsing Hua University, Hsinchu, Taiwan. Male BALB/c mice (5 weeks old and weighted 15-18 g) were purchased form National Laboratory Animal Center in Taiwan. To set up the tumor model, CT26 cells were subcutaneously inoculated into the right hind limb of the mice. Tumor sizes and body weights were measured every day for the duration of the experiment.

\section{Biodistribution of PEG-HISP by IVIS}

\section{NIR imaging of live animals}

After the tumor volumes reached $100-200 \mathrm{~mm}^{3}$, PEG-HISP $(0.3 \mathrm{mg} / \mathrm{kg}$ for IR780) was intravenously injected into the BALB/c mice. 4, 24 and 48 hours after injection, the NIR images were taken using an animal optical imaging system (IVIS Spectrum In Vivo Imaging System, PerkinElmer's IVISTM Spectrum imaging system).

\section{NIR imaging of the excised organs}

After 48 hours, the mice were sacrificed. The organs including heart, liver, lung, spleen, kidney and tumor were collected and analyzed using IVIS. The excitation and emission wavelength of IR780 were 710 $\mathrm{nm}$ and $840 \mathrm{~nm}$.

\section{In vivo photothermal therapeutic efficacy of PEG-HISP}

The treatments were started when the tumor volumes reached 50-100 $\mathrm{mm}^{3}$. PBS, free IR780, PEG-HSP or PEG-HISP were injected via tail vein into tumor-bearing mice at doses equivalent to $1 \mathrm{mg} / \mathrm{kg}$ of IR780. 24 hours after injection, the mice were irradiated at $808 \mathrm{~nm}\left(1 \mathrm{~W} / \mathrm{cm}^{2}\right)$ for 5 minutes. Three cycles of drug injection/laser irradiation were performed following by 2 additional irradiations. The temperature was recorded using a Mini thermal imager (Avio NEC Thermo Shot F30W). The tumor size was calculated using the following equation:

$$
\text { Tumor volume }=0.5 \times{\mathrm{A} \times \mathrm{B}^{2}}^{2}
$$

Where, A and B represent the largest and smallest tumor diameters respectively.

\section{In vivo compatibility of PEG-HISP}

Healthy male BALB/c mice were intravenously injected with PEG-HISP $(1 \mathrm{mg} / \mathrm{kg}$ of IR780). Saline was used as the control. After 2 weeks, mice were sacrificed to collect the blood for serum biochemistry assay. In addition, the major organs from each mouse were harvested, fixed in $4 \%$ PFA and subjected to paraffin embedding and sectioning. The sample slices were stained with hematoxylin and eosin (H\&E) then examined using a digital microscope. Effect of PEG-HISP on mouse liver function was also investigated. PBS or PEG-HISP was intravenously injected into mice and the blood was collected 14 days after injection for subsequent AST (aspartate transaminase) or ALT (alanine transaminase) analysis.

\section{Statistics}

Results of this study are presented as the mean and standard deviation of at least three independent measurements. All statistical evaluations were carried out with unpaired two-tailed Student's t-test. $p$-value of less than 0.05 was considered significant $(p<0.05$, * $\left.; p<0.01,{ }^{* *} ; p<0.001,{ }^{* * *}\right)$.

\section{Results and Discussion}

\section{Synthesis and physicochemical characterizations of PEG-HISP}

The HISP were prepared using a straightforward 
single emulsion procedure (Scheme 1) to obtain nanocomplexes containing hydrophobic core (superparamagnetic iron oxide nanoparticles (SPIONs) and IR780) bound by the hydrophilic shell (human serum albumin (HSA)). A series of designated formulations containing various ratios of HSA/IR780/SPIO were prepared and examined for the particle size. At the ratio of 150:0.5:2, the HISP at size of $118.5 \mathrm{~nm}$ was obtained and used in the following studies. Loading efficiency (L.E.) and encapsulation efficiency (E.E.) of IR780 in the HISP were determined to as $0.6 \%$ and $4.5 \%$ respectively (Table 1). In addition, the L.E. and E.E. of SPIO in the HISP were determined to as $6.6 \%$ and $12.7 \%$ respectively (Table 1). It has been previously suggested that albumin nanoparticles are subjected to rapid in vivo clearance after intravenous injection[31-34]. Jain S. et al., showed that conjugation of PEG molecules could largely improve the circulation time and therapeutic efficacy of the drug-containing albumin nanoparticles on animal tumor model[34]. To prepare PEG-HISP, the HISP was first thiolated following by conjugation of poly (ethylene glycol) (PEG)-maleimide at room temperature. Sizes of the PEG-HISP and PEG-HSP were $146.3 \mathrm{~nm}$ and $173.7 \mathrm{~nm}$ respectively (Table 2) which fit well into the range of EPR effect[35]. TEM imaging was further used to study the morphology of nanocomplexes showing that the PEG-HISP contained clusters of SPIO nanoparticles bound by the HSA (Figure 1). In vitro IR780 release from PEG-HISP in $\mathrm{DI} \mathrm{H}_{2} \mathrm{O}$ was evaluated in PBS at $37^{\circ} \mathrm{C}$ for 168 hours. Under such circumstance, cumulative release of IR780 from the PEG-HISP was less than 10\% (Figure S1). Colloidal stability of the PEG-HISP was examined at

various environments, including: $\mathrm{DI} \mathrm{H}_{2} \mathrm{O}, \mathrm{PBS}$ and $50 \%$ FBS/DMEM for designated time lengths $(0,12 \&$ 24 hours) (Figure $2 \mathrm{~A} \sim \mathrm{C}$ ). In $\mathrm{DI} \mathrm{H}_{2} \mathrm{O}$ or PBS, particle size of the PEG-HISP was mostly below $150 \mathrm{~nm}$. Successful conjugation of PEG to HISP was verified by forming PEG-HISP with larger size. In DI $\mathrm{H}_{2} \mathrm{O}$ or PBS, particle size of these PEG-HISP (HSA: PEG (1:10) or HSA: PEG (1:50)) was mostly below $150 \mathrm{~nm}$. Thus, PEG-HISP prepared using minimal amount of PEG (HSA: PEG (1:10)) was chosen for the subsequent studies. Overall particle size of the PEG-HISP was larger in 50\% FBS/DMEM medium compared to DI $\mathrm{H}_{2} \mathrm{O}$ and PBS. Long-term colloidal stability of the PEG-HISP at $4^{\circ} \mathrm{C}$ was next examined. The results show that size of the PEG-HISP was well maintained below $150 \mathrm{~nm}$ up to 1 month (Figure 2D) and no obvious aggregation was observed from the nanoparticle solutions (Figure S2) suggesting their excellent colloidal stability for long term storage.

Table 1. The encapsulated efficiency and loading efficiency of IR780 and SPIO in HISP.

\begin{tabular}{lllr}
\hline \multicolumn{2}{c}{ IR780 } & \multicolumn{2}{c}{ SPIO } \\
\hline E.E. (\%) & L.E. (\%) & E.E. (\%) & L.E. (\%) \\
\hline $4.5 \pm 0.1$ & $0.6 \pm 0.00$ & $12.7 \pm 1.9$ & $6.6 \pm 1.0$ \\
\hline
\end{tabular}

Table 2. Effect of PEGylation on the particle size of HSP and HISP.

\begin{tabular}{llll}
\hline Group & Size $(\mathrm{nm})$ & PDI & $\begin{array}{l}\text { Zeta potential } \\
(\mathrm{mV})\end{array}$ \\
\hline HSP & $144.5 \pm 11.9$ & $0.113 \pm 0.003$ & $-25.5 \pm 7.4$ \\
HISP & $118.5 \pm 5.2$ & $0.126 \pm 0.008$ & $-22.5 \pm 1.1$ \\
PEG-HSP & $173.7 \pm 21.8$ & $0.14 \pm 0.009$ & $-11.3 \pm 9.6$ \\
PEG-HISP & $146.3 \pm 40.4$ & $0.154 \pm 0.052$ & $-16.9 \pm 1.6$ \\
\hline
\end{tabular}

(C)
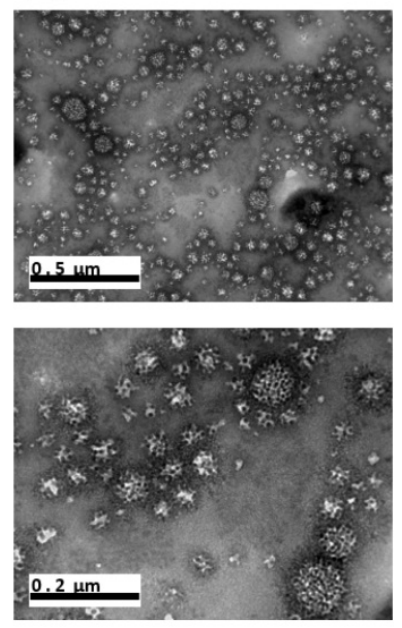

(D)

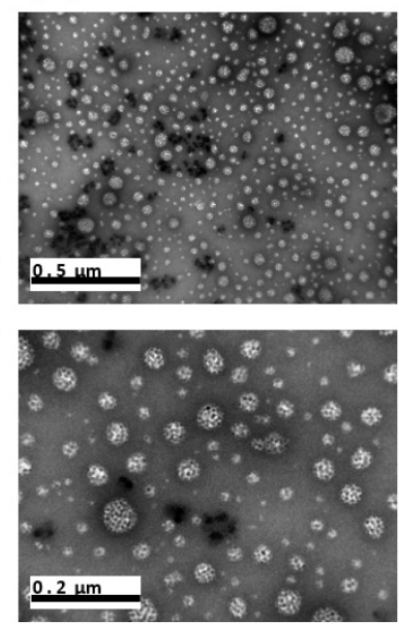

Figure 1. TEM images of (A) HSA:PEG = 1:0. (B) HSA:PEG = 1:10. (C) HSA:PEG = 1:50. (D) HSA:PEG = 1:200. Size of the scale bars are indicated in the images. 


\section{In vitro photothermal effects of PEG-HISP}

Successful encapsulation of IR780 in the fabricated nanocomplexes was confirmed using UV-Vis spectroscopic analysis (Figure 3A). Free IR780, when it was dispersed in organic solvent, showed a characteristic absorption at $780 \mathrm{~nm}$. Meanwhile, a bathochromic shift was observed from the IR780 after its encapsulation in HSA/SPIO nanoparticles (HIP, PEG-HIP, HISP or PEG-HISP) in water. This phenomenon may be attributed to the different solvent environment and hydrophobic stacking of IR780 molecules encapsulated in the nanoparticles $[36,37]$. Strong absorption at the NIR region (650-950 $\mathrm{nm}$ ) allows PEG-HISP to be used as a promising NIR photothermal agent. Photothermal effects of the IR780, HIP, HSP, PEG-HSP, HISP and PEG-HISP were studied by monitoring the laser irradiation-dependent temperature changes using a photothermal camera. After starting laser irradiation $\left(808 \mathrm{~nm}, 1 \mathrm{~W} / \mathrm{cm}^{2}\right)$, the sample temperatures were recorded every 30 seconds for 5 minutes (Figure 3B). Temperature of the solutions containing PEG-HSP, HSP, HIP or free IR780 increased less than $5{ }^{\circ} \mathrm{C}$. In contrast, much higher temperature increase $\left(\Delta \mathrm{T} \sim 14^{\circ} \mathrm{C}\right)$ was observed from the HISP or PEG-HISP solution. The results also show that PEGylation did not alter the photothermal properties of the HSP or HISP. To further evaluate the photothermal property under repeated laser irradiation, free IR780 $(20 \mu \mathrm{g} / \mathrm{mL}$, dissolved in DMSO and diluted with $\mathrm{DI} \mathrm{H}_{2} \mathrm{O}$ ) was compared with HISP. In the first three laser irradiation/cooling cycles, the temperature of free IR780 solution was significantly lower than the HISP. The ineffective photothermal effect of free IR780 might be due to its high tendency of forming aggregation in aqueous environment. In contrast, repeated photothermal effect was demonstrated from the solution containing well-dispersed HISP (Figure S3). Time-dependent cellular uptake of PEG-HISP by CT26 cells was analyzed using a flow cytometry. Higher cellular

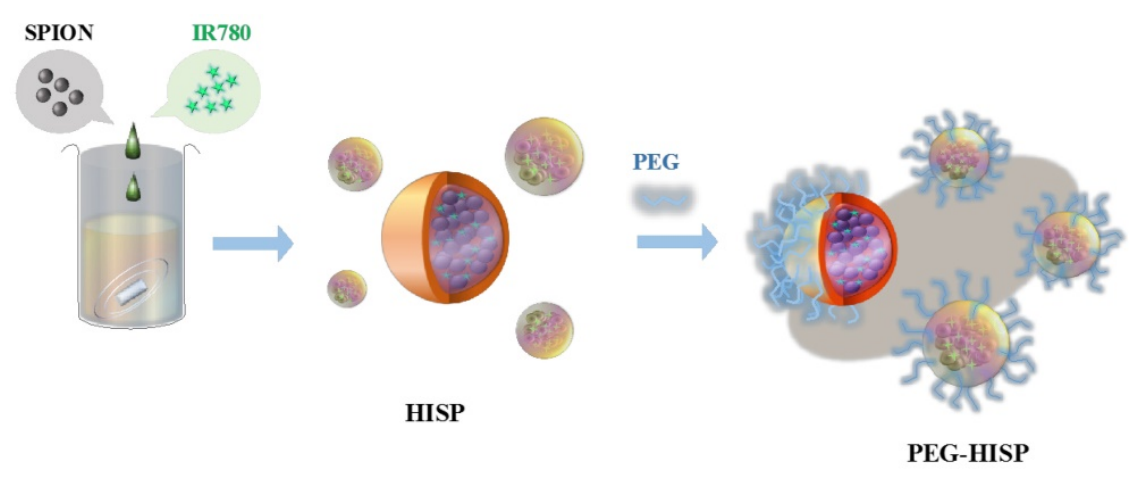

Scheme 1. Preparation of multiple functional PEG-HISP for photothermal therapy and MRI imaging. uptake was observed from free IR780 compared to HISP or PEG-HISP. IR780 showed highest uptake efficiency probably due to its low molecular weight and capability of direct diffusion through cell membrane. Cellular uptake efficiency of HISP was slightly higher than PEG-HISP. Maximal cellular uptake was observed after incubating the PEG-HISP with cells for 24 hours (Figure 4A). CT26 cancer cells were then incubated with free IR780, HISP and PEG-HISP at various IR780 concentrations for 24 hours. Negligible cytotoxicity was observed from the HISP or PEG-HISP $(0.02 \sim 10 \mu \mathrm{g} / \mathrm{mL})$ suggesting their good cell compatibility (Figure 4B). In contrast, significant cytotoxicity was observed from cells received free IR780. It is possible that the lower cytotoxicity of HISP might be due to the hindrance of IR780 within the albumin/SPIO nano-formulation. Photothermal-mediated anti-cancer effect was studied on the CT26 cells pre-fed with PEG-HSP or PEG-HISP. $808 \mathrm{~nm}$ laser was used to irradiate $\left(1.5 \mathrm{~W} / \mathrm{cm}^{2}\right)$ the cells for 1,3 or 5 minutes (Figure $4 \mathrm{C}$ ). With the increase of laser exposure, the cell viability decreased accordingly. These results suggest that PEG-HISP was not cytotoxic and exhibited effective photothermal effect.

\section{MRI applications of the magnetic HISP}

Ferrofluidic property of the albumin/SPIO nanocomplexes was observed under an external magnetic field (Figure 5A). The HISP contains SPIO, which shortens the spin-spin relaxation times (T2) of the surrounding water molecules, thus producing dose-dependent T2-weighted images (Figure 5B). Spin-spin relaxation time (T2) of the HISP was measured using a 7T MRI system. R2 relaxivity (81.6 $\mathrm{s}^{-1} \mathrm{mM}^{-1}$ ) of the HISP (Figure $5 \mathrm{C}$ ) was higher than the commercial MRI contrast agent (Feridex ${ }^{\circledR}, 63.5$ $\left.\mathrm{s}^{-1} \mathrm{mM}^{-1}\right)$. Feasibility of using the PEG-HISP as MRI contrast agent for tumor imaging was evaluated with 7T MRI system. Significant T2 tumor imaging enhancement was observed from mice injected with PEG-HISP compared to saline injection. Both the axial and coronal planes of tumors on the mouse received PEG-HISP injection showed darker MR images (Figure 6A). Significant decrease on MR signal intensity from PEG-HISP group compared to the control was confirmed by quantitative analysis of the MR images (Figure 6B). The results suggest that PEG-HISP might be utilized as a MR imaging-guided photothermal nanomaterial for cancer treatments. 
(A) $\mathrm{ddH}_{2} \mathrm{O}$

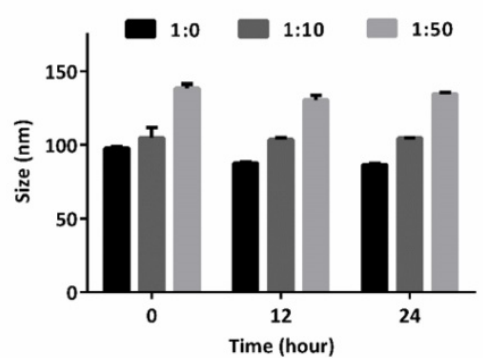

(B) $50 \%$ FBS/DMEM

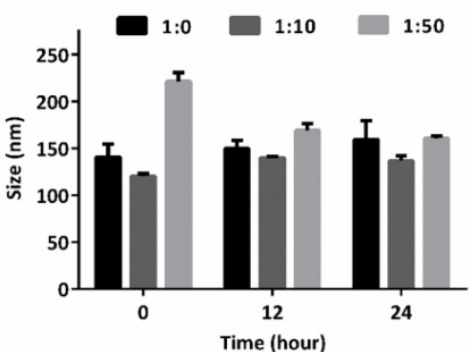

(C) PBS

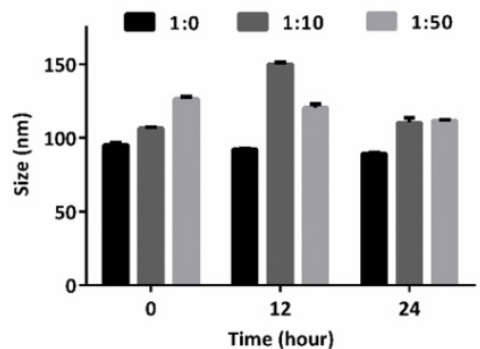

(D) PBS (long term storage)

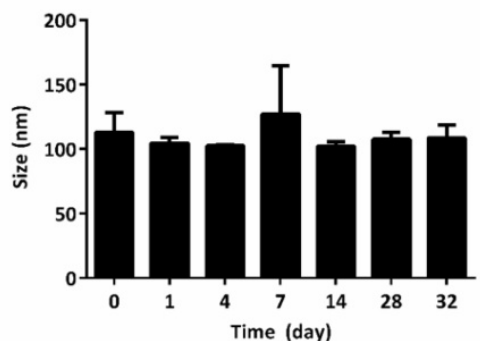

Figure 2. Colloidal stability of PEG-HISP. (A) (C) Short-term storage test in $\mathrm{DI}_{\mathrm{H}} \mathrm{O}, 50 \%$ FBS/DMEM or PBS at $37 \circ \mathrm{C}$. (D) Long-term storage test of PEG-HISP (HSA:PEG = 1:10) in PBS at 4०C. The particle size was measured after incubating the nanoparticles in the designated environments. Data represents the mean \pm S.E.; $\mathrm{n}=3$

(A)

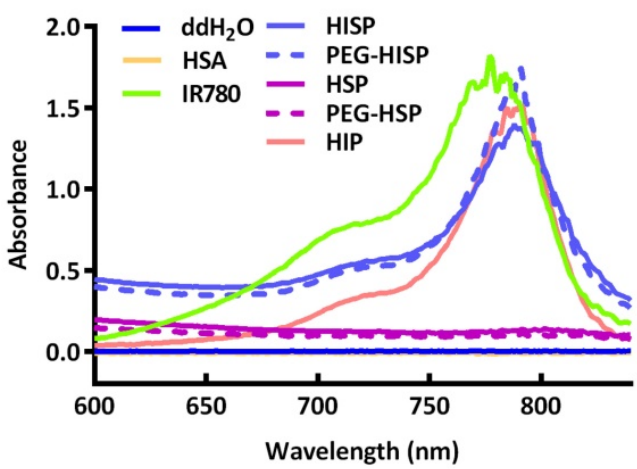

(B)

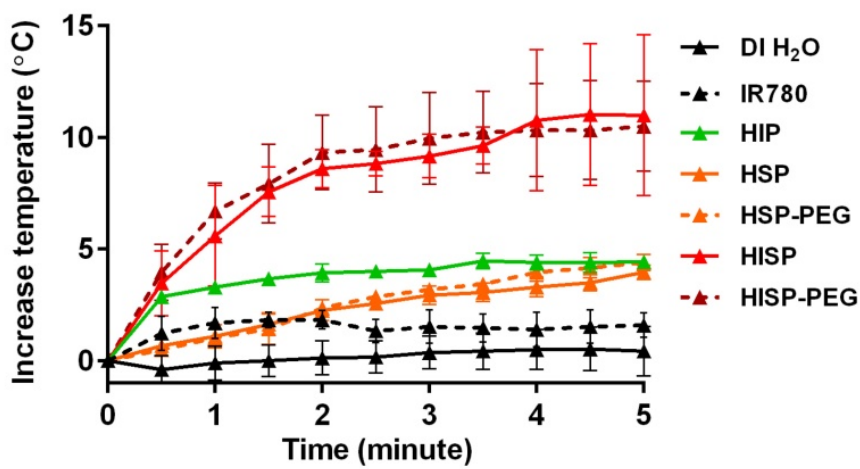

Figure 3. (A) UV-visible spectrum of HSA, free IR780, HIP, HSP, PEG-HSP, HISP and PEG-HISP. The characteristic NIR absorption range was detected from free IR780 or PEG-HISP sample solutions. (B) Photothermal properties of PEG-HISP. The effect of NIR irradiation duration on heating the solutions of free IR780, HIP, HSP, PEG-HSP, HISP or PEG-HISP. Data represents the mean \pm S.E.; $n=3$.

(A)

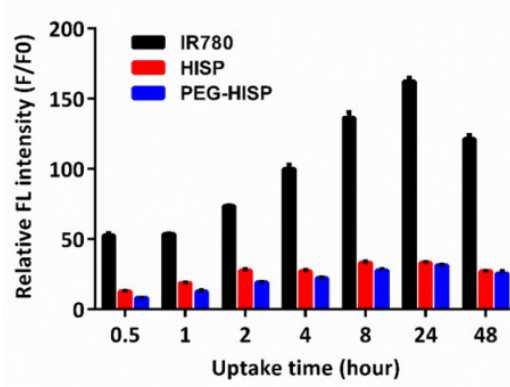

(B)

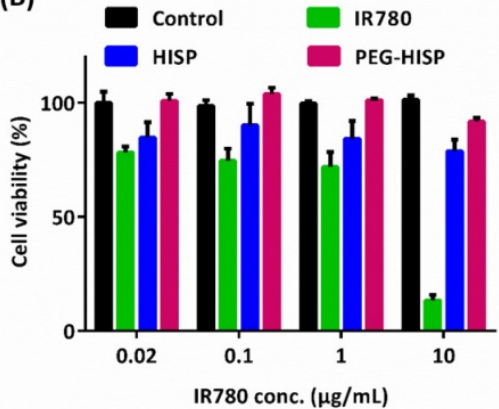

(C)

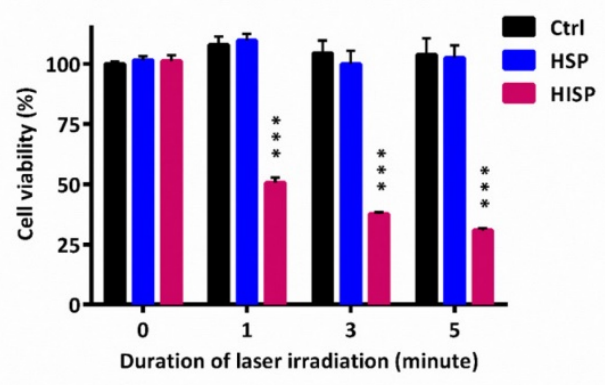

Figure 4. (A) Time course analysis of free IR780, HISP, and PEG-HISP taken by CT26 cancer cells. (B) Effect of IR780 concentration on the cytotoxicity assay of free IR780, HISP or PEG-HISP on CT26 cancer cells. (C) Effect of NIR (808 nm) irradiation on the viability of CT26 cancer cells. Data represents the mean \pm S.E.; $n=3$. 


\section{Tumor accumulation, photothermal therapy and biocompatibility of PEG-HISP on animal colon cancer model}

Accumulation of the PEG-HISP in CT26 tumor was studied by tracking the fluorescence signal of IR780 using IVIS (Figure 7). CT26 tumor-bearing mice were intravenously injected with PBS, free IR780 or PEG-HISP and all the mice were kept alive during the period of test. Fluorescence signals of the tumors glowed with time on mice received free IR780 or PEG-HISP. Twenty-four or forty-eight hours after injections, much higher IR780 accumulation in tumors was observed from the mice injected PEG-HISP compared to free IR780. After 48 hours, the mice were sacrificed to harvest organs including liver, lung, heart, spleen, kidney and tumor for IVIS detection. Similarly, higher IR780 accumulation was observed from the PEG-HISP group, which was consistent to the live IVIS observation. To further study the photothermal therapeutic efficacy, PBS, free IR-780, PEG-HSP or PEG-HISP was intravenously injected to the CT26 tumor-bearing mice. Twenty-four hours after injections, the mice were irradiated by a laser $\left(808 \mathrm{~nm}, 1.0 \mathrm{~W} / \mathrm{cm}^{2}\right)$ for $5 \mathrm{~min}$. The drug injection/laser irradiation cycle was repeated for 3 times. Within laser irradiation, the PEG-HISP groups showed much higher heating effect than free IR780 and PEG-HSP groups as observed using a thermal camera (Figure S4). The slight photo-thermal effect of PEG-HSP might be due to the presence of SPIO in the nanocomplexes[38-40]. Meanwhile, tumor temperature of the PEG-HISP groups elevated much higher than that of free IR780 or PEG-HSP groups. As a result, significant tumor shrinkage was observed from the mice injected with PEG-HISP combing with NIR irradiation (Figure 8). To assess the potential toxicity of PEG-HISP in tissue level, mice were sacrificed 2 weeks after injections to harvest the major organs (heart, liver, spleen, lung \& kidney). These organs were paraffin-embedded, sliced and stained with hematoxylin and eosin (H\&E) reagents for histological analysis. No noticeable tissue damage or infiltration of inflammatory cells was observed from any of the major organs (Figure 9). Potential liver toxicity of PEG-HISP was also investigated to show that both AST (aspartate transaminase) and ALT (alanine transaminase) levels were not significantly different between PEG-HISP-treated mice and control mice (Figure S5).
(A)

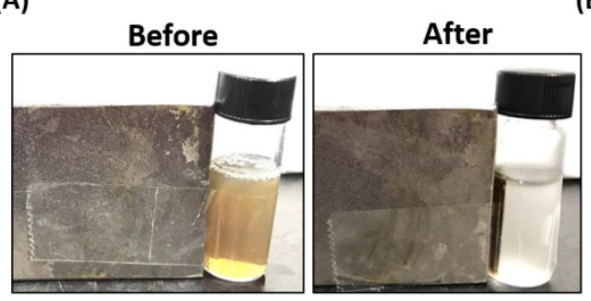

(B)

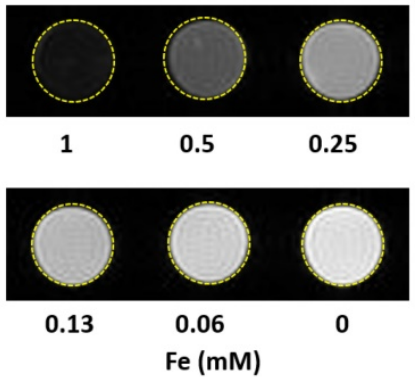

(C)

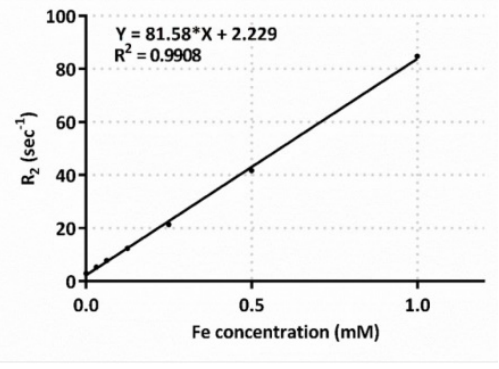

Figure 5. Magnetic properties measurements. (A) Albumin/SPIO nanocomplexes dispersed in $\mathrm{DI} \mathrm{H}_{2} \mathrm{O}$ before (left) and after (right) magnetic attraction. (B) In vitro T2-weighted MR images: various concentrations of HISP in DI $\mathrm{H}_{2} \mathrm{O}$. (C) T2 relaxivity measurement of HISP.

(A)

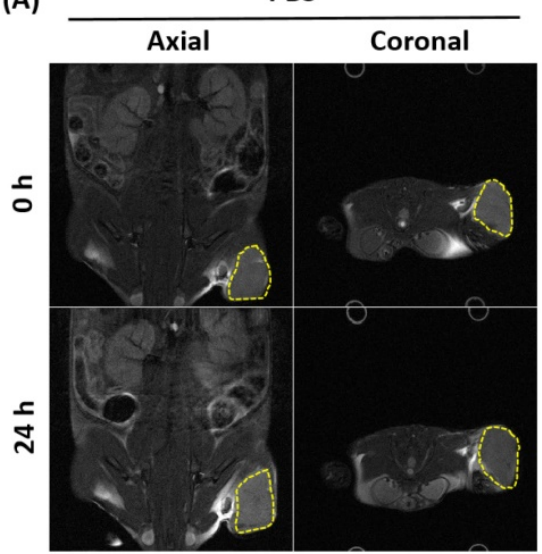

PEG-HISP

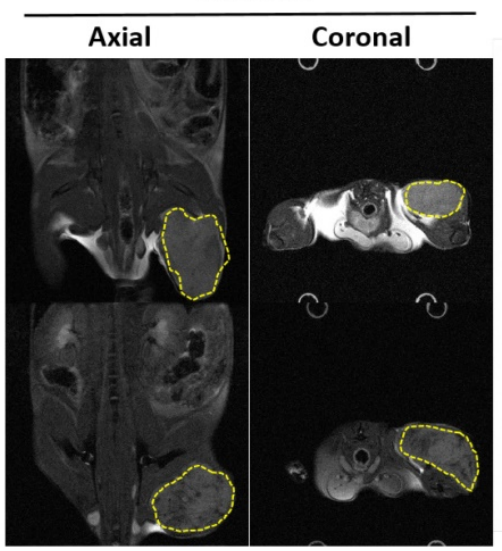

(B)

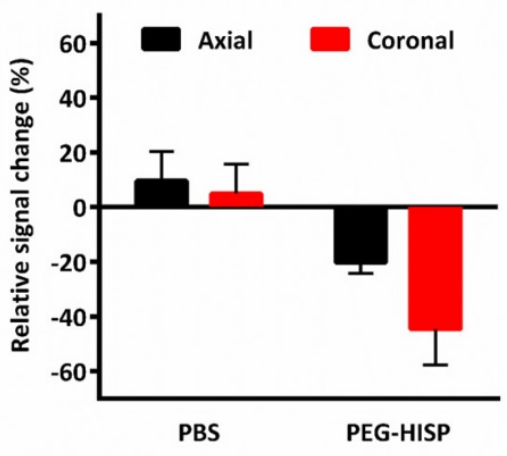

Figure 6. T2-weighted MR images of the tumor at 0 hour or 24 hours after the injections of PBS or PEG-HISP. (A) MR imaging contrast (dark spots) enhancement were found in both axial and coronal tumor sections (yellow dashed circle) on mice received PEG-HISP injections. (B) Quantitative analysis of the acquired MR images from mice received PBS or PEG-HISP injecitons. 
(A)

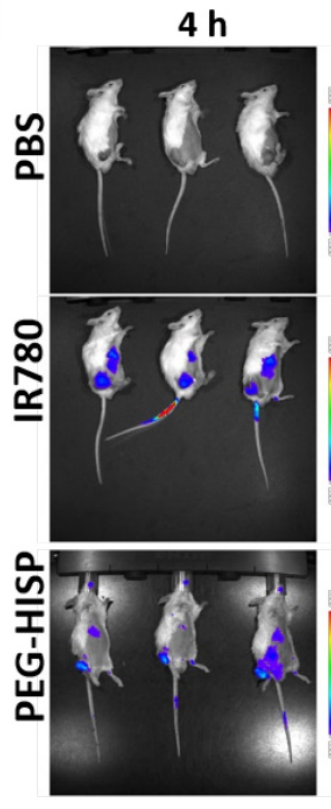

$24 \mathrm{~h}$

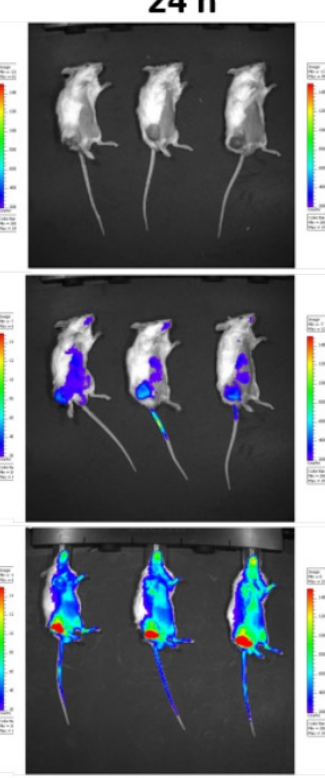

$48 \mathrm{~h}$

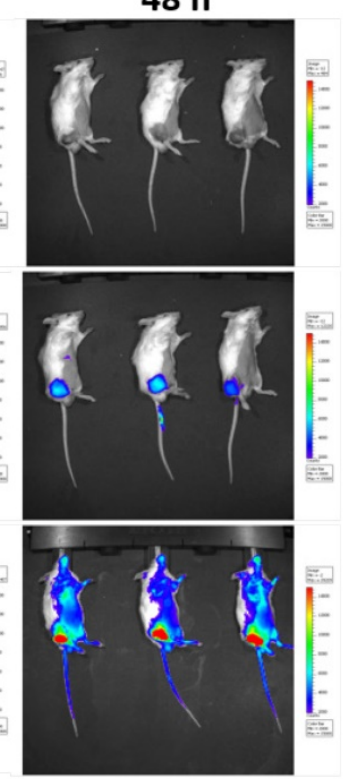

(B)
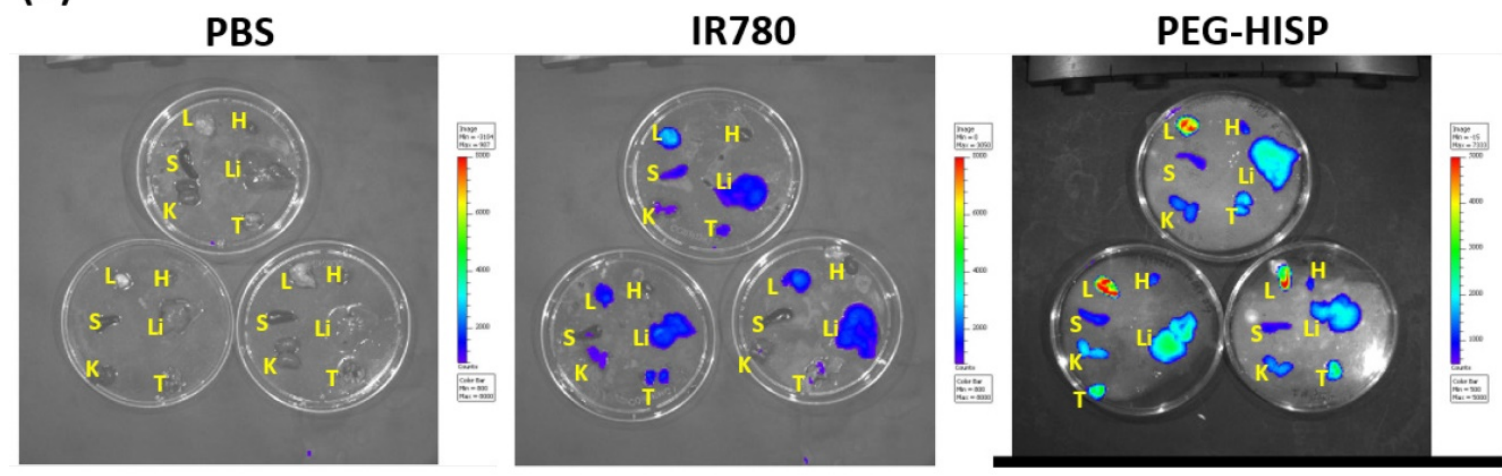

Figure 7. Biodistribution analysis of the intravenously injected free IR780 or PEG-HISP on CT26 tumor-bearing mice using IVIS. (A) After received the predesignated injections, the mice were anesthetized and live images were acquired. (b) 48 hours after injection, the mice were sacrificed to harvest the organs for taking images. L: lung; H: heart; S: spleen; Li: liver; K: kidney; T: tumor.

Albumin-based nano-formulation has been proposed to improve the solubility, photo-stability and in vivo therapeutic efficacy of photothermal dyes[37, 41, 42]. In this study, incorporation of SPIO further afford the IR780-albumin nano-formulation with capabilities on MRI and magnetic targeted drug delivery. Generally, albumin-SPIO nanocomplexes are most commonly prepared by deposition of albumin on the surface of SPIO via covalent or noncovalent interactions[43, 44]. Here, we explored the feasibility of using single emulation method to prepare nanoclusters of albumin/SPIO co-encapsulating with IR780. In vivo photothermal therapeutic efficacy and MR imaging were successfully demonstrated from the proposed PEG-HISP nanoplatform.

\section{Conclusion}

The PEG-HISP was successfully prepared by utilizing FDA-approved materials (SPIO and HSA) to deliver hydrophobic IR780. The resultant nanocomplexes displayed favorable properties for anti-cancer drug delivery, including: (1) Small particle size (<150 nm); (2) Good colloidal stability (no aggregation at $4^{\circ} \mathrm{C}$ up to 1 month) and (3) Excellent photothermal effect and stability. The magnetic PEG-HSP exhibited comparable T2 relaxivity to the commercial MRI T2 contrast agent. Clear tumor MR imaging was demonstrated from the tumor-bearing mice injected with the PEG-HISP. Photothermal therapeutic effects of the PEG-HISP were demonstrated on a murine colon cancer model. Taking together, our findings suggest PEG-HISP is a potential nanotheranostic system for cancer treatment. In the future, specific tumor-targeting moieties could be conjugated to the PEG-HISP to enhance its tumor-specific accumulation thus further promoting the anti-cancer efficacy. 
(A)

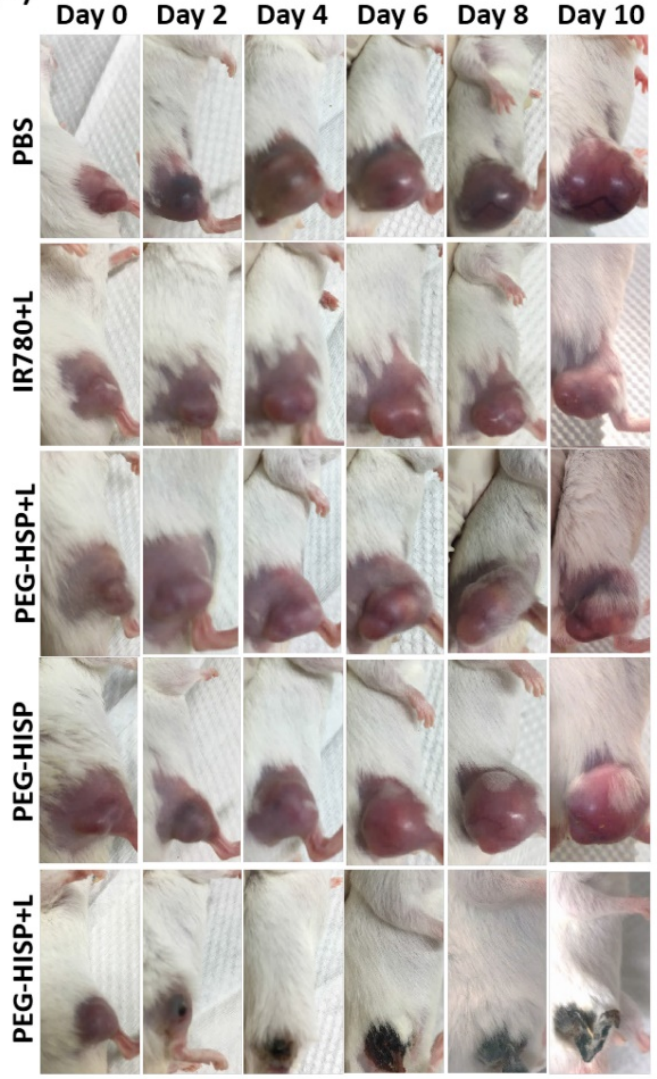

(B)

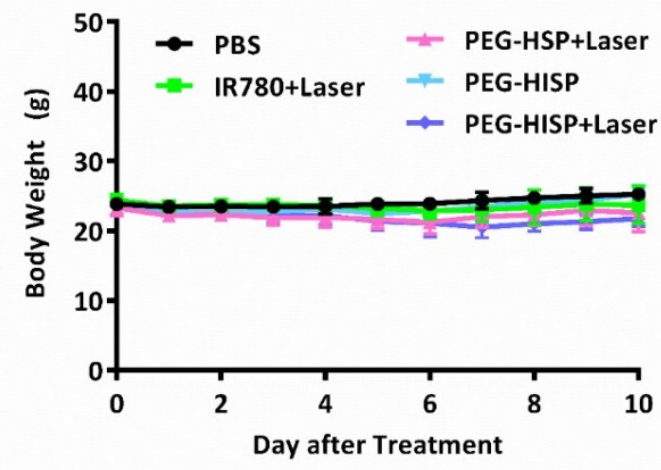

(C)

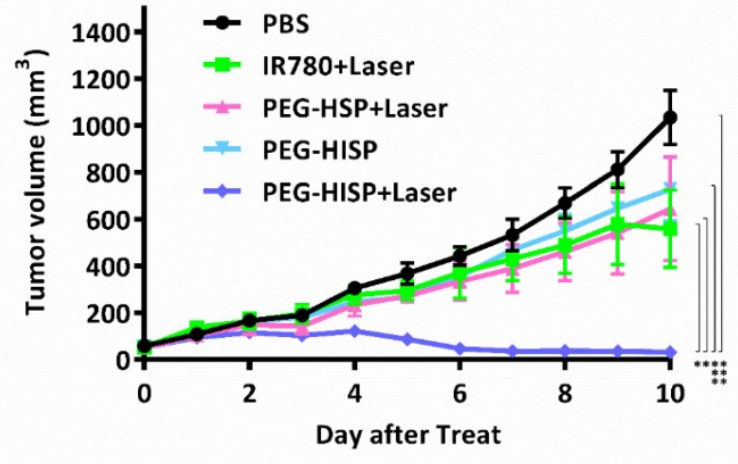

Figure 8. In vivo phototherapeutic efficacy of PEG-HISP. (A) Mice received the designated drug injections with or without NIR irradiation were imaged. (B) Time course study of mouse body weight. (C) Time course study of tumor volume changes. Data represents the mean \pm S.E.; $n=5$.

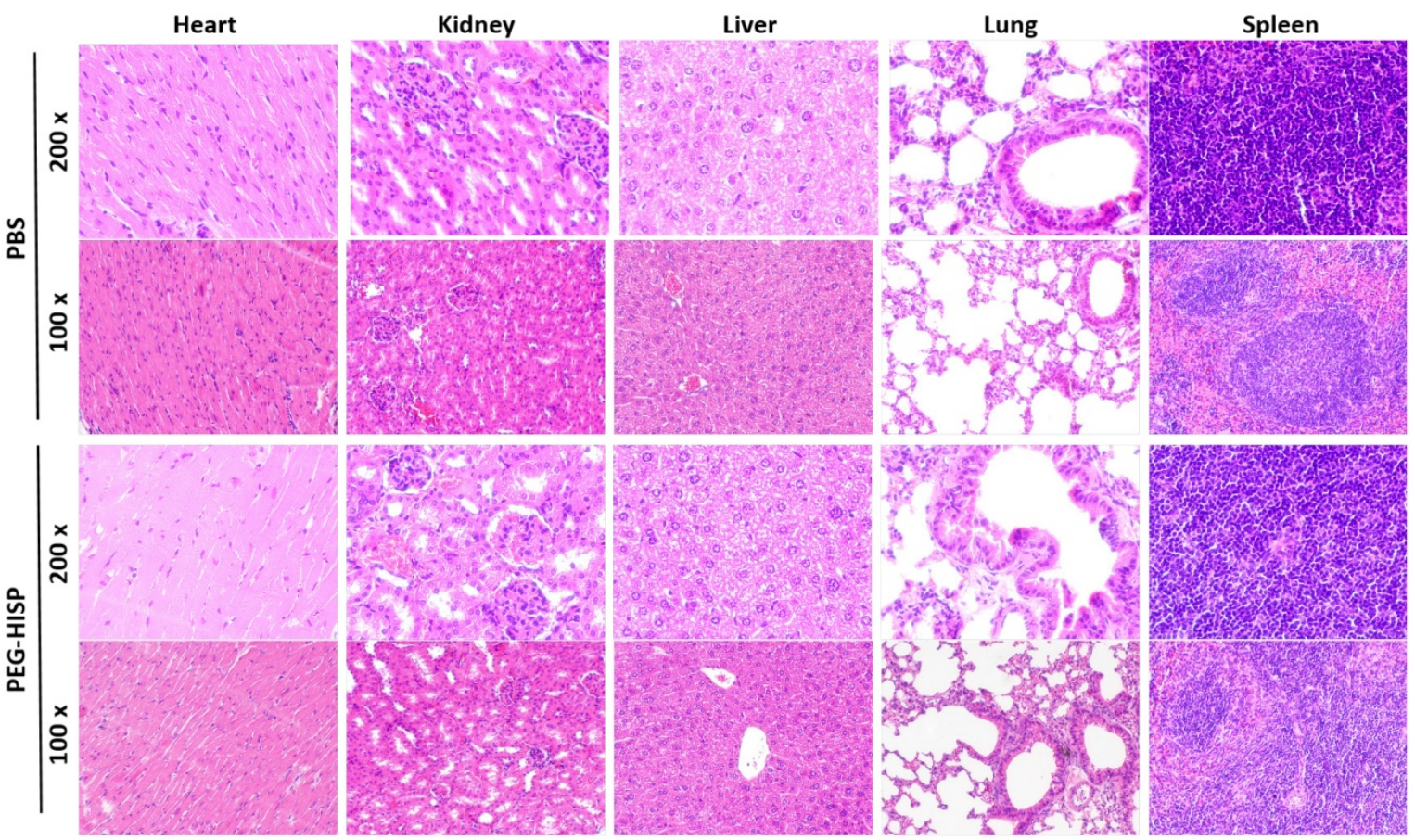

Figure 9. Histological analysis of organs excised from mice received PBS or PEG-HISP injections. 


\section{Supplementary Material}

Supplementary figures.

http://www.ntno.org/v02p0106s1.pdf

\section{Acknowledgment}

This research was financially supported by Ministry of Science and Technology of Taiwan (MOST 105-2119-M-007-023-) and National Tsing Hua University (106J00X9K6 / 106N522CE1). We thank to Ms. C.-Y. Chien of Ministry of Science and Technology (National Taiwan University) for the assistance in TEM experiments and to 7T animal MRI Core Lab of the Neurobiology and Cognitive Science Center, National Taiwan University for technical and facility supports.

\section{Competing Interests}

The authors have declared that no competing interest exists.

\section{References}

1. Adam Chicheł JS, Magda Kubaszewska, Marek Kanikowski. Hyperthermia description of a method and a review of clinical applications. Rep Pract Oncol Radiother. 2007; 12: 267-75.

2. Chu KF, Dupuy DE. Thermal ablation of tumours: biological mechanisms and advances in therapy. Nat Rev Cancer. 2014; 14: 199-208.

3. van der Zee J. Heating the patient: a promising approach? Annals of oncology : official journal of the European Society for Medical Oncology. 2002; 13: 1173-84.

4. Ahmed K, Tabuchi Y, Kondo T. Hyperthermia: an effective strategy to induce apoptosis in cancer cells. Apoptosis. 2015; 20: 1411-9.

5. Perez-Hernandez M, Del Pino P, Mitchell SG, Moros M, Stepien G, Pelaz B, et al. Dissecting the molecular mechanism of apoptosis during photothermal therapy using gold nanoprisms. ACS Nano. 2015; 9: 52-61.

6. Melamed JR, Edelstein RS, Day ES. Elucidating the fundamental mechanisms of cell death triggered by photothermal therapy. ACS Nano. 2015; 9: 6-11.

7. Shi Y, Liu M, Deng F, Zeng G, Wan Q, Zhang X, et al. Recent progress and development on polymeric nanomaterials for photothermal therapy: a brief overview. J Mater Chem B. 2017; 5: 194-206.

8. Liang C, Diao S, Wang C, Gong H, Liu T, Hong GS, et al. Tumor metastasis inhibition by imaging-guided photothermal therapy with single-walled carbon nanotubes. Adv Mater. 2014; 26: 5646-5652.

9. Deng H, Zhong Y, Du M, Liu Q, Fan Z, Dai F, et al. Theranostic self-assembly structure of gold nanoparticles for NIR photothermal therapy and X-Ray computed tomography imaging. Theranostics. 2014; 4: 904-18.

10. Weissleder R. A clearer vision for in vivo imaging. Nat Biotechnol. 2001; 19: 316-7.

11. Yuan A, Wu JH, Tang XL, Zhao LL, Xu F, Hu YQ. Application of near-infrared dyes for tumor imaging, photothermal, and photodynamic therapies. J Pharm Sci-Us. 2013; 102: 6-28.

12. Luo SL, Zhang EL, Su YP, Cheng TM, Shi CM. A review of NIR dyes in cancer targeting and imaging. Biomaterials. 2011; 32: 7127-38.

13. Kim J, Kim J, Jeong C, Kim WJ. Synergistic nanomedicine by combined gene and photothermal therapy. Adv Drug Deliv Rev. 2016; 98: 99-112.

14. Son S, Nam J, Kim J, Kim S, Kim WJ. i-motif-driven Au nanomachines in programmed siRNA delivery for gene-silencing and photothermal ablation. ACS Nano. 2014; 8: 5574-84

15. Liu Y, Kang N, Lv J, Zhou ZJ, Zhao QL, Ma LC, et al. Deep photoacoustic/luminescence/magnetic resonance multimodal imaging in living subjects using high-efficiency upconversion nanocomposites. Adv Mater. 2016; 28: 6411-6419.

16. Liu Y, Yang Y, Sun M, Cui M, Fu Y, Lin Y, et al. Highly specific noninvasive photoacoustic and positron emission tomography of brain plaque with functionalized croconium dye labeled by a radiotracer. Chem Sci. 2017; 8: 2710-6.

17. Kang N, Liu Y, Zhou YM, Wang D, Chen C, Ye SF, et al. Phase and size control of core-shell upconversion nanocrystals light up deep dual luminescence imaging and CT in vivo. Adv Healthc Mater. 2016; 5: 1356-63.

18. Zhang C, Wang S, Xiao J, Tan X, Zhu Y, Su Y, et al. Sentinel lymph node mapping by a near-infrared fluorescent heptamethine dye. Biomaterials. 2010; 31: 1911-7.
19. Yue $\mathrm{C}$, Liu $\mathrm{P}$, Zheng $\mathrm{M}$, Zhao $\mathrm{P}$, Wang $\mathrm{Y}$, Ma Y, et al. IR-780 dye loaded tumor targeting theranostic nanoparticles for NIR imaging and photothermal therapy. Biomaterials. 2013; 34: 6853-61.

20. Guo F, Yu M, Wang J, Tan F, Li N. Smart IR780 Theranostic nanocarrier for tumor-specific therapy: hyperthermia-mediated bubble-generating and folate-targeted liposomes. ACS applied materials \& interfaces. 2015; 7: 20556-67.

21. Wang KK, Zhang YF, Wang J, Yuan A, Sun MJ, Wu JH, et al. Self-assembled IR780-loaded transferrin nanoparticles as an imaging, targeting and PDT/PTT agent for cancer therapy. Sci Rep-Uk. 2016; 6.

22. Shi S, Liu Y, Chen Y, Zhang Z, Ding Y, Wu Z, et al. Versatile pH-response micelles with high cell-penetrating helical diblock copolymers for photoacoustic imaging guided synergistic chemo-photothermal therapy. Theranostics. 2016; 6: 2170-82.

23. Cortes J, Saura C. Nanoparticle albumin-bound (nab (TM))-paclitaxel: improving efficacy and tolerability by targeted drug delivery in metastatic breast cancer. Ejc Suppl. 2010; 8: 1-10.

24. Yardley DA. nab-Paclitaxel mechanisms of action and delivery. J Control Release. 2013; 170: 365-72.

25. Maeda H, Fang J, Inutsuka T, Kitamoto Y. Vascular permeability enhancement in solid tumor: various factors, mechanisms involved and its implications. Int Immunopharmacol. 2003; 3: 319-28.

26. Xie J, Wang JH, Niu G, Huang J, Chen K, Li XG, et al. Human serum albumin coated iron oxide nanoparticles for efficient cell labeling. Chem Commun. 2010; 46: 433-5.

27. Zhou Z, Song J, Tian R, Yang Z, Yu G, Lin L, et al. Activatable singlet oxygen generation from lipid hydroperoxide nanoparticles for cancer therapy. Angew Chem Int Ed Engl. 2017; 56: 6492-6.

28. Fan $\mathrm{CH}$, Cheng $\mathrm{YH}$, Ting $\mathrm{CY}$, Ho YJ, Hsu PH, Liu HL, et al. Ultrasound/magnetic targeting with SPIO-DOX-microbubble complex for image-guided drug delivery in brain tumors. Theranostics. 2016; 6: 1542-56.

29. Xie J, Chen K, Huang J, Lee S, Wang JH, Gao J, et al. PET/NIRF/MRI triple functional iron oxide nanoparticles. Biomaterials. 2010; 31: 3016-22.

30. Huang RY, Chiang PH, Hsiao WC, Chuang CC, Chang CW. Redox-sensitive polymer/SPIO nanocomplexes for efficient magnetofection and MR imaging of human cancer cells. Langmuir. 2015; 31: 6523-31.

31. Allemann E, Gurny R, Doelker E. Drug-loaded nanoparticles - preparation methods and drug targeting issues. Eur J Pharm Biopharm. 1993; 39: 173-91.

32. Stolnik S, Illum L, Davis SS. Long circulating microparticulate drug carriers. Adv Drug Deliver Rev. 2012; 64: 290-301.

33. Katre NV. The conjugation of proteins with polyethylene-glycol and other polymers - altering properties of proteins to enhance their therapeutic potential. Adv Drug Deliver Rev. 1993; 10: 91-114.

34. Jain S, Mathur R, Das M, Swarnakar NK, Mishra AK. Synthesis, pharmacoscintigraphic evaluation and antitumor efficacy of methotrexate-loaded, folate-conjugated, stealth albumin nanoparticles. Nanomedicine-Uk. 2011; 6: 1733-54.

35. Blanco E, Shen H, Ferrari M. Principles of nanoparticle design for overcoming biological barriers to drug delivery. Nat Biotechnol. 2015; 33: 941-51.

36. Li S, Johnson J, Peck A, Xie Q. Near infrared fluorescent imaging of brain tumor with IR780 dye incorporated phospholipid nanoparticles. Journal of translational medicine. 2017; 15: 18

37. Jiang $\mathrm{C}$, Cheng $\mathrm{H}$, Yuan A, Tang $\mathrm{X}, \mathrm{Wu}$ J, Hu Y. Hydrophobic IR780 encapsulated in biodegradable human serum albumin nanoparticles for photothermal and photodynamic therapy. Acta Biomater. 2015; 14: 61-9.

38. Chu M, Shao Y, Peng J, Dai X, Li H, Wu Q, et al. Near-infrared laser light mediated cancer therapy by photothermal effect of $\mathrm{Fe} 3 \mathrm{O} 4$ magnetic nanoparticles. Biomaterials. 2013; 34: 4078-88.

39. Yu TJ, Li PH, Tseng TW, Chen YC. Multifunctional Fe3O4/alumina core/shell MNPs as photothermal agents for targeted hyperthermia of nosocomial and antibiotic-resistant bacteria. Nanomedicine-Uk. 2011; 6: 1353-63.

40. Liao MY, Lai PS, Yu HP, Lin HP, Huang CC. Innovative ligand-assisted synthesis of NIR-activated iron oxide for cancer theranostics. Chem Commun (Camb). 2012; 48: 5319-21

41. Chen Q, Wang C, Zhan Z, He W, Cheng Z, Li Y, et al. Near-infrared dye bound albumin with separated imaging and therapy wavelength channels for imaging-guided photothermal therapy. Biomaterials. 2014; 35: 8206-14.

42. Rong P, Huang P, Liu Z, Lin J, Jin A, Ma Y, et al. Protein-based photothermal theranostics for imaging-guided cancer therapy. Nanoscale. 2015; 7: 16330-6.

43. Xie J, Wang J, Niu G, Huang J, Chen K, Li X, et al. Human serum albumin coated iron oxide nanoparticles for efficient cell labeling. Chem Commun (Camb). 2010; 46: 433-5.

44. Zaloga J, Pottler $M$, Leitinger G, Friedrich RP, Almer G, Lyer $S$, et al. Pharmaceutical formulation of HSA hybrid coated iron oxide nanoparticles for magnetic drug targeting. Eur J Pharm Biopharm. 2016; 101: 152-62. 